\title{
Associations between Type 2 Diabetes Mellitus and Arterial Stiffness: A Prospective Analysis Based on the Maine-Syracuse Study
}

\author{
Merrill F. Elias ${ }^{a, b} \quad$ Georgina E. Crichton ${ }^{c}$ Peter J. Dearborn ${ }^{a}$ \\ Michael A. Robbins ${ }^{a, b} \quad$ Walter P. Abhayaratna ${ }^{d}$ \\ a Department of Psychology and ${ }^{b}$ Graduate School of Biomedical Sciences and Engineering, \\ University of Maine, Orono, ME, USA; ${ }^{C}$ Alliance for Research in Exercise, Nutrition and \\ Activity (ARENA), Sansom Institute for Health Research, University of South Australia, \\ Adelaide, SA, and ${ }^{\mathrm{d} C o l l e g e}$ of Medicine, Biology, and Environment, Australian National \\ University, Canberra, ACT, Australia
}

\section{Keywords}

Arterial stiffness - Type 2 diabetes mellitus · Pulse wave velocity

\begin{abstract}
Background: The aim of this study was to investigate prospective associations between type 2 diabetes mellitus status and the gold standard non-invasive method for ascertaining arterial stiffness, carotid femoral pulse wave velocity. Methods: The prospective analysis employed 508 community-dwelling participants (mean age 61 years, 60\% women) from the Maine-Syracuse Longitudinal Study. Pulse wave velocity at wave 7 (2006-2010) was compared between those with type 2 diabetes mellitus at wave $6(2001-2006)(n=52)$ and non-diabetics at wave $6(n=456)$, with adjustment for demographic factors, cardiovascular risk factors and lifestyle- and pulse wave velocity-related factors. Results: Type 2 diabetes mellitus status was associated with a significantly higher pulse wave velocity ( $12.5 \pm 0.36$ vs. $10.4 \pm 0.12 \mathrm{~m} / \mathrm{s})$. Multivariate adjustment for other cardiovascular risk factors and lifestyle- and pulse wave velocity-related variables did not attenuate the findings. The risk of an elevated pulse wave velocity ( $\geq 12 \mathrm{~m} / \mathrm{s}$ ) was over 9 times higher for those with uncontrolled type 2 diabetes mellitus than for those without diabetes (OR 9.14, 95\% CI 3.23-25.9, $p<0.001$ ). Conclusions: Type 2 diabetes mellitus, particularly if uncontrolled, is significantly associated with risk of arterial stiffness later in life. Effective management of diabetes mellitus is an important element of protection from arterial stiffness.

(c) 2017 S. Karger AG, Basel
\end{abstract}




\section{Introduction}

Arterial stiffness is a major risk factor for myocardial infarction, stroke, end-stage renal disease and other cardiovascular diseases (CVDs) [1-3]. Mortality rates from CVD in 20032006 were approximately 1.7 times higher in US adults diagnosed with diabetes mellitus than in adults without diabetes [4]. It is now recognized that measures of central arterial function are better and more useful predictors of vascular health outcomes than measures of traditional blood pressure (BP) [5], and pulse wave velocity (PWV) is now considered the gold standard non-invasive method for measuring aortic stiffness and, more generally, arterial stiffness [6].

Prenner and Chirinos [7] provide a recent thorough review of the literature on arterial stiffness in diabetes mellitus. Increased arterial stiffness is an independent predictor of mortality and cardiovascular events both in people with diabetes and in the general population [5, 8]. As summarised in this review, the majority of studies demonstrating associations between arterial stiffness and diabetes mellitus have been cross-sectional [7]. A prospective study by Cruickshank et al. [5] in the UK of mortality in type 2 diabetes mellitus (T2DM) showed that those with T2DM who died over a mean follow-up period of 10 years were older, smoked more, and had PWV and systolic pressures that were higher on average by $2.6 \mathrm{~m} / \mathrm{s}$ and $10 \mathrm{~mm} \mathrm{Hg}$, respectively, than those who survived. PWV was higher in T2DM subjects than in controls, and those who died had higher baseline PWV on average for any level of baseline systolic pressure [5]. Other prospective studies have shown associations between pulse pressure and coronary heart disease and stroke [9], all-cause mortality in individuals with impaired fasting glucose [10] and CVD mortality in subjects with T2DM but not in those without diabetes [11]. Johansen et al. [12] showed that each yearly increase in haemoglobin $\mathrm{A} 1 \mathrm{c}(\mathrm{HbA} 1 \mathrm{c})$ of $0.1 \%$ points was associated with a $0.24 \mathrm{~m} / \mathrm{s}$ higher PWV over a 7 -year period.

Few studies have examined the relationship between T2DM duration and aortic stiffness, taking into account the influence of other lifestyle and cardiovascular factors. Using data collected from participants of the Maine-Syracuse Longitudinal Study (MSLS), 2 hypotheses were advanced: (1) T2DM will be prospectively associated with increased mean arterial stiffness (as inferred by PWV) over a 4- to 5-year period when adjusted for multiple cardiovascular risk factors and lifestyle- and PWV-related variables; and (2) the magnitude of association between T2DM and PWV for individuals with well-controlled glycaemic levels will be less than that seen for persons with suboptimally controlled diabetes.

\section{Materials and Methods}

\section{Participants}

The MSLS was a community-based study of cardiovascular risk factors and cognitive functioning in adults from the Central New York area $[13,14]$. It employed a time-lagged longitudinal-cohort design with new subjects recruited into the study every 5 years. Persons diagnosed with psychiatric illness and alcoholism were excluded at recruitment.

Comprehensive data on CVD risk factors and disease were first collected at wave 6 (baseline of this study). PWV data were obtained for the first time at wave 7. This allowed for a prospective design in which T2DM status at wave 6 (2001) was used to predict PWV at wave 7 (2006) with adjustment for demographic variables and cardiovascular risk factors (mean time between waves: $4.7 \pm 0.6$ years). Eight-hundred twentytwo subjects were invited to the laboratory for testing at wave 7. Six-hundred nine participants returned and completed PWV data collection. Participants were excluded for the following reasons: having missing data for health variables $(n=53)$, history of acute stroke $(n=28)$, probable dementia $(n=8)$, undertaking renal dialysis treatment $(n=5)$, inability to read English $(n=1)$, and prior alcohol abuse $(n=1)$, leaving a sample of 508 study participants. None of the study participants had type 1 diabetes. 
Table 1. Demographic, health and PWV factors at waves 6 and 7 according to T2DM status a at wave 6 in the MSLS sample $(n=508)$

\begin{tabular}{|c|c|c|c|c|c|c|c|c|c|}
\hline \multirow[t]{2}{*}{ Variable } & \multicolumn{3}{|l|}{ Wave 6} & \multicolumn{3}{|l|}{ Wave 7} & \multicolumn{3}{|c|}{ Change wave 6 - wave 7} \\
\hline & $\begin{array}{l}\text { no diabetes } \\
(n=456)\end{array}$ & $\begin{array}{l}\text { diabetes } \\
(n=52)\end{array}$ & $p^{\mathrm{b}}$ & $\begin{array}{l}\text { no diabetes } \\
(n=456)\end{array}$ & $\begin{array}{l}\text { diabetes } \\
(n=52)\end{array}$ & $p^{\mathrm{b}}$ & $\begin{array}{l}\text { no diabetes } \\
(n=456)\end{array}$ & $\begin{array}{l}\text { diabetes } \\
(n=52)\end{array}$ & $p$ \\
\hline \multicolumn{10}{|l|}{ Demographics } \\
\hline Age, years & $60.8 \pm 11.8$ & $63.0 \pm 9.3$ & 0.2 & $65.5 \pm 11.8$ & $67.7 \pm 9.4$ & 0.2 & 4.7 & 4.7 & 0.8 \\
\hline Education, years & $14.9 \pm 2.7$ & $13.6 \pm 2.3$ & 0.001 & $14.9 \pm 2.7$ & $13.6 \pm 2.3$ & 0.001 & 0 & 0 & - \\
\hline Smoking, cigarettes/day & $1.3 \pm 5.2$ & $1.9 \pm 6.9$ & 0.5 & $0.9 \pm 3.8$ & $1.3 \pm 6.0$ & 0.6 & -0.4 & -0.7 & 0.6 \\
\hline BMI & $28.9 \pm 5.5$ & $32.9 \pm 7.8$ & $<0.001$ & $29.1 \pm 5.8$ & $32.8 \pm 9.4$ & $<0.001$ & 0.2 & -0.1 & 0.6 \\
\hline PA, MET-h/week & $21.6 \pm 25.9$ & $14.1 \pm 17.1$ & 0.04 & $19.5 \pm 25.0$ & $12.8 \pm 27.5$ & 0.1 & -0.6 & -0.6 & 0.9 \\
\hline Total cholesterol, mg/dL & $205 \pm 38.9$ & $187 \pm 44.0$ & 0.002 & $191 \pm 39.5$ & $167 \pm 38.0$ & $<0.001$ & -13.8 & -19.6 & 0.3 \\
\hline HDL cholesterol, mg/dL & $55.8 \pm 15.9$ & $46.6 \pm 10.7$ & $<0.001$ & $54.0 \pm 15.4$ & $44.8 \pm 11.6$ & $<0.001$ & -1.8 & -1.9 & 0.9 \\
\hline LDL cholesterol, mg/dL & $124 \pm 32.8$ & $106 \pm 36.3$ & $<0.001$ & $115 \pm 32.9$ & $93.3 \pm 27.8$ & $<0.001$ & -9.0 & -11.8 & 0.6 \\
\hline Systolic BP, mmHg & $128.0 \pm 21.0$ & $137.8 \pm 21.4$ & 0.002 & $129.1 \pm 20.4$ & $137.4 \pm 19.0$ & 0.005 & 1.1 & -0.4 & 0.6 \\
\hline Diastolic BP, mmHg & $69.7 \pm 9.9$ & $70.8 \pm 8.9$ & 0.4 & $77.0 \pm 9.7$ & $76.8 \pm 9.6$ & 0.9 & 7.3 & 6.0 & 0.3 \\
\hline $\mathrm{FBG}, \mathrm{mg} / \mathrm{dL}$ & $91.6 \pm 9.8$ & $153.9 \pm 54.7$ & $<0.001$ & $93.8 \pm 13.5$ & $127.4 \pm 38.1$ & $<0.001$ & 2.3 & -26.5 & $<0.001$ \\
\hline Triglycerides, mg/dL & $132 \pm 110$ & $177 \pm 113$ & 0.006 & $112 \pm 64.2$ & $160 \pm 156$ & $<0.001$ & -20.1 & -16.6 & 0.8 \\
\hline $\mathrm{CRP}^{\mathrm{c}}, \mathrm{mg} / \mathrm{L}$ & $0.40 \pm 0.48$ & $0.53 \pm 0.49$ & 0.06 & - & - & - & - & - & - \\
\hline Alcohol, g/week & $35.9 \pm 60.7$ & $15.7 \pm 35.1$ & 0.02 & $41.8 \pm 69.7$ & $20.7 \pm 50.4$ & 0.03 & 5.9 & 4.9 & 0.9 \\
\hline \multicolumn{10}{|l|}{$P W V$ variables } \\
\hline $\mathrm{PWV}, \mathrm{m} / \mathrm{s}$ & - & - & - & $10.3 \pm 2.9$ & $13.0 \pm 3.4$ & $<0.001$ & - & - & - \\
\hline $\mathrm{MAP}, \mathrm{mm} \mathrm{Hg}$ & $89.1 \pm 12.5$ & $93.2 \pm 11.7$ & 0.03 & $94.3 \pm 12.0$ & $96.9 \pm 11.7$ & 0.1 & 5.2 & 4.1 & 0.5 \\
\hline Weight, kg & $81.3 \pm 18.1$ & $93.4 \pm 24.7$ & $<0.001$ & $81.7 \pm 18.6$ & $89.6 \pm 20.0$ & 0.004 & 0.3 & -2.5 & 0.01 \\
\hline Height, cm & $167 \pm 10.1$ & $167 \pm 11.7$ & 0.4 & $167 \pm 10.1$ & $167 \pm 11.7$ & 0.4 & 0 & 0 & 0 \\
\hline Heart rate, bpm & $66.3 \pm 9.5$ & $71.4 \pm 13.0$ & 0.001 & $59.4 \pm 8.9$ & $65.1 \pm 10.4$ & $<0.001$ & -6.9 & -6.3 & 0.6 \\
\hline \multicolumn{10}{|l|}{ Comorbidities $^{\mathrm{d}}$} \\
\hline $\mathrm{CVD}^{\mathrm{e}}, \%$ & 8.6 & 19.2 & 0.01 & 12.5 & 25.0 & 0.01 & 3.9 & 5.8 & 0.5 \\
\hline Hypertensionf, \% & 53.9 & 80.8 & $<0.001$ & 61.0 & 82.7 & 0.002 & 7.1 & 1.9 & 0.3 \\
\hline Obesityg, $\%$ & 35.8 & 61.5 & $<0.001$ & 35.8 & 55.8 & 0.005 & 0 & -5.7 & 0.3 \\
\hline
\end{tabular}

Values for wave 6 and wave 7 are given as means \pm standard deviations unless otherwise indicated. Values for change (wave 6 minus wave 7 ) are given as "mean change" for demographics and PWV variables, and as “\% change” for comorbidities. BMI, body mass index; BP, blood pressure; CRP, C-reactive protein; CVD, cardiovascular disease; FBG, fasting blood glucose; HDL, high-density lipoprotein; Hcy, homocysteine; LDL, low-density lipoprotein; MAP, mean arterial pressure; MET, metabolic equivalent; PA, physical activity; PWV, pulse wave velocity; T2DM, type 2 diabetes mellitus. ${ }^{\mathrm{a}} \mathrm{T} 2 \mathrm{DM}$ was defined as a fasting glucose level of $\geq 126 \mathrm{mg} / \mathrm{dL}$ or being treated with anti-diabetic medication. ${ }^{\mathrm{b}} p$ values were obtained by analysis of variance for continuous variables or the $\chi^{2}$ test for categorical variables. ${ }^{c}$ PWV was not measured at wave 6 , and CRP was not measured at wave 7 due to reduced funding. ${ }^{\mathrm{d}}$ Change in $\%$ values from wave 6 to 7 are expressed as wave 7 minus wave 6 values. e CVD was based upon self-reported history of coronary artery disease, myocardial infarction, congestive heart failure, transient ischemic attack or angina pectoris, confirmed by medical records. ${ }^{\mathrm{f}}$ Hypertension was defined as BP $\geq 140 / 90$ mm Hg or being treated with anti-hypertensive medication. g Obesity was defined as BMI $\geq 30$.

The proportion of persons in the diabetes group at wave 6 (10.1\%; Table 1 ) is the same as the proportion of those with T2DM in the Framingham Heart Study of diabetes and cognitive performance (10.3\%) [15].

Acute stroke was defined as a focal neurological deficit of acute onset persisting more than $24 \mathrm{~h}$ and was based on self-report and confirmed by a record review indicating a diagnosis of acute stroke. Clinical diagnoses of dementia were determined by a team including 2 neuropsychologists, a social psychologist and a geriatric physician using cognitive data, medical records and criteria established by the National Institute of Neurological and Communicative Diseases and Stroke/Alzheimer's Disease and Related Disorders Association (NINCDS-ADRDA) criteria [16]. Recently, the diagnostic decisions were confirmed using the ICD-10 guidelines [17].

This study was conducted according to the guidelines established by the Declaration of Helsinki, and all procedures and analyses were approved by the University of Maine and State University of New York (SUNY) Health Sciences Center.

\section{$B P$ and $P W V$ Assessment}

Automated BP measures (GE DINAMAP 100DPC-120XEN, GE Healthcare) were taken in the right arm 5 times each in recumbent, standing and sitting position after a supine rest for $15 \mathrm{~min}$, and the 15 values were averaged for systolic BP and diastolic BP, as dictated by the MSLS protocol. Mean arterial pressure (MAP) was calculated using the following: diastolic $\mathrm{BP}+(1 / 3 \times$ pulse pressure $)$.

Following a rest, carotid femoral PWV was assessed non-invasively in the supine position following the SphygmoCor ${ }^{\circledR}$ protocol (AtCor Medical, Sydney, NSW, Australia). A minimum of 2 but sometimes 3 
Elias et al.: Associations between Type 2 Diabetes Mellitus and Arterial Stiffness: A Prospective Analysis Based on the Maine-Syracuse Study

serial assessments of PWV were obtained in order to obtain a single assessment that best met SphygmoCor criteria for a record of acceptable quality. Data are reported as the results of that single best assessment. The PWV technician was trained to a high level of proficiency by a cardiologist, who as part of the training supervised the procedure. Electrocardiogram-gated carotid and femoral waveforms were recorded using applanation tonometry. Carotid-femoral path length was measured as the difference between the surface distances joining (1) the suprasternal notch, the umbilicus and the femoral pulse and (2) the suprasternal notch and the carotid pulse. Carotid-femoral transit time was estimated in 8-10 sequential femoral and carotid waveforms as the average time difference between the onset of the femoral and carotid waveforms. The foot of the pulse wave was identified using the intersecting tangent method. The distance measurements were entered into the software in millimetres. PWV was calculated as the carotid-femoral path length divided by the carotid-femoral transit time and expressed in $\mathrm{m} / \mathrm{s}$. This is an established, widely employed, non-invasive and reproducible method to determine arterial stiffness [6]. The coefficient of variation $(1.79 \%)$ for serial measurements of PWV in our laboratory indicates high reproducibility of the PWV measurements [13].

\section{Predictor Variables}

T2DM (wave 6) was defined by self-report of diabetes, confirmed by glucose levels at wave 6 and 7, or drug treatment for diabetes. Controlled fasting diabetes was defined as having glucose levels of 80-130 mg/ $\mathrm{dL}$ and controlled non-fasting diabetes as glucose levels of $<180 \mathrm{mg} / \mathrm{dL}$ [18]. Duration of diabetes at wave 7 was calculated based on the initial date of determination subtracted from the date of participation at wave 7. Fasting glucose levels at wave 6 were employed in separate analyses. Treatment as usual was conducted by the patient's physician with treatment information entered into the participant's protocol for our use. HbA1c levels were obtained by the metabolic clinic and/or the participant's physician when essential to a diagnosis, but individual values were not available to our study.

Diabetic participants at baseline (wave 6) and wave 7 were taking 1 or more of 15 diabetic medications with a range of $0-3$ being taken in combination. The following classes of hypertensive drugs were used, alone or in combination: angiotensin-converting enzyme inhibitors, angiotensin II receptor blockers, calcium channel blockers, $\alpha$ blockers and $\beta$ blockers. HbA1c levels were obtained by the metabolic clinic and/or the patient's physician when essential to a diagnosis, but individual values were not made available to our study.

\section{Covariates}

Demographic, socioeconomic and lifestyle characteristics were obtained from the Nutrition and Health Questionnaire [19, 20]. Data obtained included smoking history, marital status and medical history. Physical activity was measured with the Nurses' Health Study Activity Questionnaire, a validated measure of time spent engaging in various physical activities [21]. Education level was obtained through self-report and ranged from 4 to 20 years. Dietary intake was assessed using the Nutrition and Health Questionnaire, including a widely validated food frequency questionnaire $[19,20]$.

Among diabetic individuals, duration of diabetic medication, current prescription status (diabetic medication) and insulin-specific treatment status were all not related to PWV ( $p \geq 0.18$ for all) and, thus, were not included in our regression models as covariates had to be related both to the predictor and outcome variables. Obesity was defined as a body mass index (BMI; $\mathrm{kg} / \mathrm{m}^{2}$ ) of $\geq 30$, and CVD was based upon self-reported history of coronary artery disease, myocardial infarction, congestive heart failure, transient ischemic attack or angina pectoris, confirmed by medical records. Standard assay methods were employed [22] to obtain fasting plasma glucose $(\mathrm{mg} / \mathrm{dL})$, total cholesterol $(\mathrm{mg} / \mathrm{dL})$, low-density lipoprotein cholesterol $(\mathrm{mg} / \mathrm{dL})$, high-density lipoprotein cholesterol $(\mathrm{mg} / \mathrm{dL})$, triglycerides $(\mathrm{mg} / \mathrm{dL})$, C-reactive protein (CRP; $\mathrm{mg} / \mathrm{L})$ and plasma homocysteine $(\mu \mathrm{mol} / \mathrm{L})$ following an overnight fast. Blood sampling methods and assays have been fully described previously [22].

\section{Statistical Analyses}

Participant demographics, health and dietary variables and BP measures were compared according to T2DM (yes/no) at wave 6. Independent-samples $t$ tests were used for continuous variables and the $\chi^{2}$ test for categorical variables for the analyses involving demographic variables.

Analysis of variance (categorical regression analyses) was used to relate T2DM with PWV using 3 covariate models. The following models were employed to adjust for confounding:

- $\quad$ Model 1: basic model: age (years) + gender + education (years) + ethnicity;

- $\quad$ Model 2: PWV model: Basic + heart rate $(\mathrm{bpm})+\mathrm{MAP}(\mathrm{mm} \mathrm{Hg})+$ height $(\mathrm{cm})+$ weight $(\mathrm{kg})$; 
Elias et al.: Associations between Type 2 Diabetes Mellitus and Arterial Stiffness: A Prospective Analysis Based on the Maine-Syracuse Study

- $\quad$ Model 3: full model: PWV model + smoking (cigarettes/day), physical activity (metabolic equivalent-h/ week), plasma homocysteine ( $\mu \mathrm{mol} / \mathrm{L})$, alcohol intake (g/day) and C-reactive protein $(\mathrm{mg} / \mathrm{L})$.

The rule for including covariates was that they had to be related to the predictor and the outcome (potential confounders) or be clinically relevant, e.g., cholesterol levels. Additional and alternative models were employed in sensitivity analyses reported later in this paper.

In addition, logistic regression was used to determine the likelihood of having elevated PWV, defined as $\geq 12 \mathrm{~m} / \mathrm{s}$ [23], using the same models as described above. There is no formally agreed upon level for high PWV, but normative data $[23,24]$ suggest a cut-off score of $12 \mathrm{~m} / \mathrm{s}$ as clearly in the range of high PWV. The non-diabetes group served as the reference category. Analysis of variance was also used to compare PWV between controlled and uncontrolled glycaemic levels and hypertension in persons with diabetes.

Statistical analyses were performed with PASW for Windows ${ }^{\circledR}$ version 21.0 software (formerly SPSS Statistics Inc., Chicago, IL, USA); $p<0.05$ for 2 -tailed tests was considered statistically significant.

Preliminary Tests of Interactions

Preliminary to the selection of the final models, tests of interactions between T2DM and age and sex were performed, as were tests of interactions of T2DM with BP, hypertension and CVD. None of the interactions reached statistical significance (all $p>0.10$ ).

\section{Results}

Table 1 shows the demographic and health-related variables at waves 6 and 7 according to T2DM status at wave 6, the difference scores for these variables and associated $p$ values. Ten percent of the sample had diabetes at wave 6 , of which $48 \%$ were female. The mean duration in which diabetes had been diagnosed prior to wave 6 was $2.7 \pm 4.2$ years. Of diabetic individuals, $81 \%$ were on diabetes medication at wave 6 , and $73 \%$ were also on BP medication. Change in covariates from waves 6 to 7 was observed in diabetics for 3 variables, i.e., fasting glucose (decreased), plasma homocysteine (increased) and weight (decreased) (all $p<0.05)$, and a marginal increase in diabetic medication use was found ( $p=0.08)$. The prevalence of diabetes increased to $15 \%$ at wave 7 .

Compared to those without diabetes, diabetic individuals at wave 6 exhibited significantly higher BMI, systolic BP, plasma homocysteine, fasting plasma glucose and triglycerides. Individuals with diabetes had significantly lower cholesterol levels, performed less physical activity and consumed less alcohol than people free from diabetes. These trends at wave 6 were similar at wave 7 .

Figures 1 and 2 show systolic BP and fasting plasma glucose levels for the 3 groups of participants, i.e., no diabetes, controlled diabetes and uncontrolled diabetes, at waves 6 and 7.

\section{Main Effects of Diabetes}

PWV was significantly higher in those with T2DM at wave 6 (mean PWV $12.7 \pm 0.36 \mathrm{~m} / \mathrm{s}$ ) than in those without diabetes (mean PWV $10.4 \pm 0.12 \mathrm{~m} / \mathrm{s})(p<0.001)$ for the basic model. This significant difference remained with the addition of PWV-related variables, lifestyle factors and cardiovascular risk factors (model 3: with T2DM: mean PWV $12.3 \pm 0.33 \mathrm{~m} / \mathrm{s}$ vs. without diabetes: mean PWV $10.4 \pm 0.11 \mathrm{~m} / \mathrm{s} ; p<0.001)$.

Table 2 shows the odds ratios (OR) and 95\% confidence intervals (CI) associated with elevated PWV ( $\geq 12 \mathrm{~m} / \mathrm{s}$ ) at wave 7 , according to T2DM status at wave 6. Study participants without diabetes served as the reference group for this analysis, and this group was compared with T2DM with well-controlled and suboptimally controlled glycaemic levels. Individuals with uncontrolled diabetes had over an 8-fold greater risk of elevated PWV than individuals without diabetes (OR 8.40, 95\% CI 3.46-20.4, $p<0.001$, basic model). With the addition of PWV-related variables and cardiovascular risk factors, this association between uncontrolled 
Fig. 1. Systolic blood pressure at waves 6 and 7 according to diabetes status at wave 6.
Fig. 2. Fasting plasma glucose at waves 6 and 7 according to diabetes status at wave 6 .
Elias et al.: Associations between Type 2 Diabetes Mellitus and Arterial Stiffness: A Prospective Analysis Based on the Maine-Syracuse Study
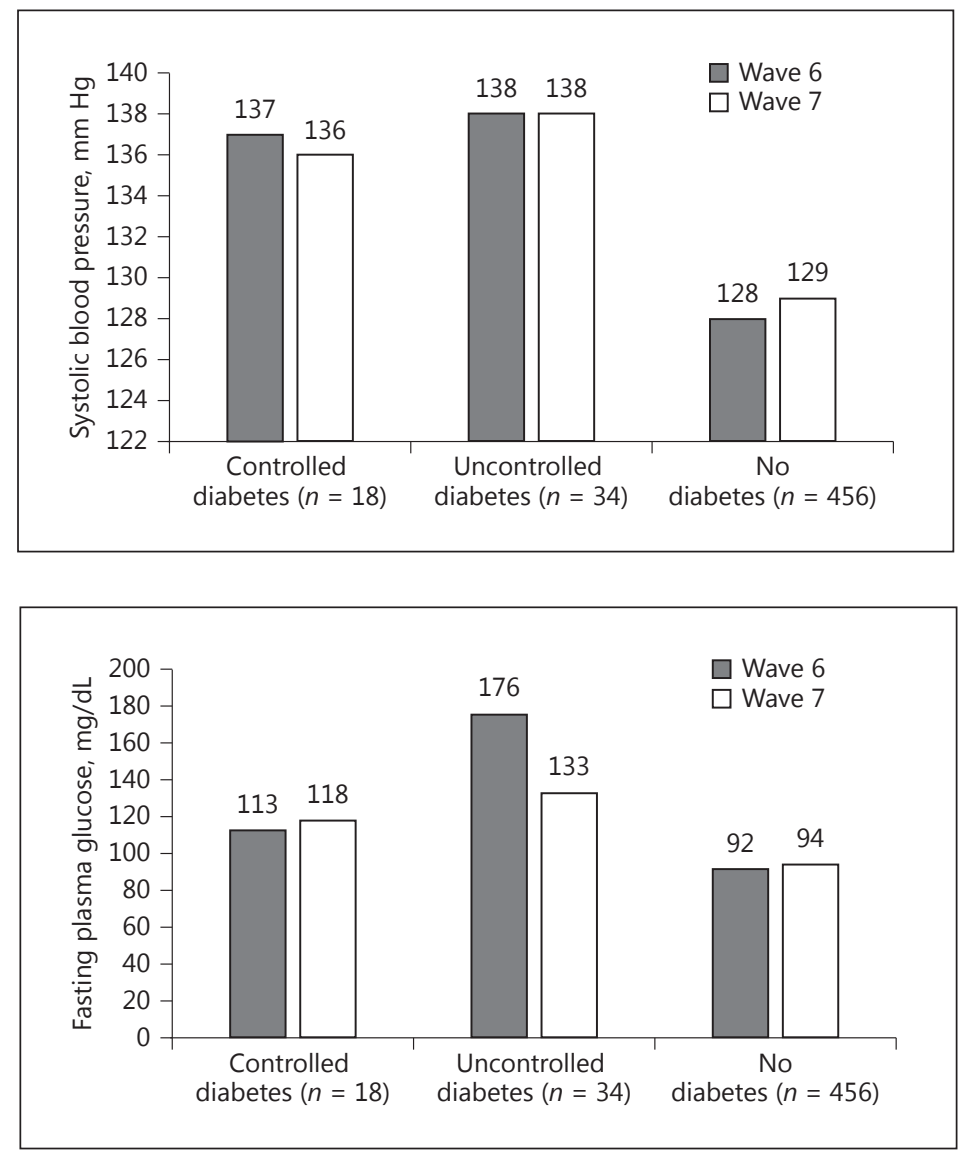

T2DM and PWV remained (OR 9.14, 95\% CI 3.23-25.9, $p<0.001$, model 3). Controlled T2DM subjects also had a significantly higher PWV than those without diabetes (OR 3.20, 95\% CI $0.99-10.3, p=0.05$, model 2 ). This was no longer statistically significant in the fully extended model ( $p=0.06$ ), but results were in the same direction (OR 3.13, 95\% CI 0.96-10.3).

In a final step, we examined the relationship between fasting plasma glucose and PWV in a subset of individuals $(n=477)$, including diabetic individuals who were permitted to fast via our institutional protocol. Fasting plasma glucose was positively associated with PWV, with statistical adjustment for the variables in model 3 (b $=0.019,95 \%$ CI $0.011-0.027, p<$ 0.001 ) and in the lower-order models (both $p<0.001$; data not shown). The study protocol did not permit diagnosed diabetics to fast without their physician's approval.

\section{Sensitivity Analyses}

The PWV-related variables taken from wave 7 in model 2 (heart rate, height, weight and MAP) were taken from wave 6 in a sensitivity analysis, and the results remained unchanged. Moreover, when weight and height (from wave 7) were replaced by waist circumference and then BMI (from wave 7), no change in the results was observed. Further, replacing MAP (at wave 7) with systolic and diastolic BP (taken at wave 7) [25] and hypertension (yes/no) in 3 separate analyses did not alter the results, nor did adding treatment with anti-hypertensive medication (yes/no) to the third model.

Adjusting for cardiovascular risk factors at wave 7 rather than wave 6 made no difference in the findings (model 3: with T2DM: mean PWV $12.4 \pm 0.39 \mathrm{~m} / \mathrm{s}$ vs. without diabetes: mean PWV $10.3 \pm 0.12 \mathrm{~m} / \mathrm{s}, p<0.001)$. 
Elias et al.: Associations between Type 2 Diabetes Mellitus and Arterial Stiffness: A Prospective Analysis Based on the Maine-Syracuse Study

Table 2. OR associated with high PWV ( $\geq 12 \mathrm{~m} / \mathrm{s})$ at wave 7 according to T2DM status at wave $6(n=508)$

\begin{tabular}{lllcc}
\hline Model & T2DM status at wave 6 & \multicolumn{3}{l}{ PWV at wave $7 \geq 12 \mathrm{~m} / \mathrm{s}$} \\
\cline { 3 - 4 } & & OR & $95 \% \mathrm{CI}$ & $p$ value \\
\hline Basic model $^{\mathrm{a}}$ & Diabetes uncontrolled $(n=34,6.5 \%)$ & 8.40 & $3.46-20.4$ & $<0.001$ \\
& Diabetes controlled $(n=18,3.6 \%)$ & 3.22 & $1.06-9.78$ & 0.04 \\
& No diabetes $(n=456,89.9 \%)$ & 1.00 & & \\
\hline Model 2 $^{\mathrm{b}}$ & Diabetes uncontrolled & 6.73 & $2.53-17.9$ & $<0.001$ \\
& Diabetes controlled & 3.20 & $0.99-10.3$ & 0.05 \\
& No diabetes & 1.00 & & \\
\hline Model 3 $^{\mathrm{c}}$ & Diabetes uncontrolled & 9.14 & $3.23-25.9$ & $<0.001$ \\
& Diabetes controlled & 3.13 & $0.96-10.3$ & 0.06 \\
& No diabetes & 1.00 & & \\
\hline
\end{tabular}

CI, confidence interval; OR, odds ratio; PWV, pulse wave velocity; T2DM, type 2 diabetes mellitus. ${ }^{\text {a }}$ Basic

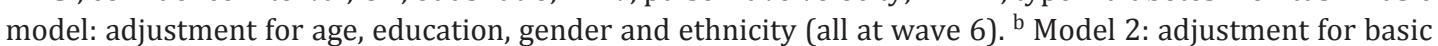
covariates + height, weight, heart rate and mean arterial pressure (all at wave 7). ${ }^{\mathrm{c}}$ Model 3: adjustment for model 2 covariates + smoking, physical activity, homocysteine, alcohol and C-reactive protein (from waves 6 and 7).

In additional sensitivity analyses, total cholesterol and then high-density lipoprotein and low-density lipoprotein cholesterol and triglycerides were added to model 3 (in separate analyses). Results remained unchanged (PWV remained significantly higher in those with T2DM than in those without, $p<0.001$ for both).

Among diabetic individuals at wave $7(n=83)$, the duration of diagnosis in years (mean 5.12, SD 4.494) was related to higher PWV (wave 7) adjusting for basic demographics (model $1: \mathrm{b}=0.252,95 \%$ CI $0.132-0.373, p<0.001$ ), PWV-related variables (model 2: $\mathrm{b}=0.240,95 \%$ CI $0.123-0.357, p<0.001$ ) and extended risk factor covariates (model $3: \mathrm{b}=0.197,95 \% \mathrm{CI}$ $0.073-0.322, p=0.002)$. Duration of diagnosis accounted for $12.6 \%$ of the variance $\left(R^{2}\right)$ of PWV in model 3 (model $R^{2}=0.45$ ).

\section{Attrition}

Participants who completed both waves 6 and $7(n=508)$ were compared with those who did not return for wave 7 testing $(n=464)$. Those who dropped out were older, had higher systolic and diastolic BP, higher plasma homocysteine and lower high-density lipoprotein cholesterol (all $p<0.05$ ). A slightly higher proportion of individuals who did not complete wave 7 were diagnosed with T2DM, CVD or hypertension (all $p<0.05$ ) compared to completers.

\section{Discussion}

Arterial stiffness is a powerful independent risk factor of cardiovascular events and mortality $[3,26]$. PWV is the gold standard non-invasive measure of arterial stiffness [6]. It is thus very important to know if there are prospective relations between diabetes and PWV because cross-sectional studies provide no information about the direction of this relationship. Very few long-term studies considering duration of diabetes and controlled versus non-controlled diabetes have been done using a prospective design. 
Data from the present study confirmed our 2 hypotheses: (1) T2DM did prospectively associate with increased mean arterial stiffness (as inferred by PWV) over a 4- to 5-year period when adjusted for multiple cardiovascular risk factors and lifestyle- and PWV-related variables; and (2) the magnitude of association between T2DM and PWV for individuals with well-controlled glycaemic levels was less than that seen for persons with suboptimally controlled diabetes. The risk of elevated PWV associated with diabetes was statistically significant after adjustment for cardiovascular risk factors, physical activity, smoking, CRP and alcohol intake. Further, the findings remained with additional adjustment for use of antihypertensive medications. The finding that control for diabetes is associated with lower PWV, and by inference less arterial stiffness, is particularly important because the means for control of diabetes are readily available and often successful.

Consistent with the literature [7, 27], duration of time in years between first detection of T2DM and wave 7 PWV assessment was positively correlated with magnitude of PWV. This finding supports the work of others [27-29]. Agnoletti et al. [28] examined 618 T2DM patients and showed that across increasing tertiles of T2DM duration PWV increased significantly, while glycaemic control and renal function worsened [28]. T2DM duration was independently associated with PWV, explaining about $4 \%$ of PWV variability from low to high tertiles [28]. Positive results have, therefore, been demonstrated with adjustment for CVD variables, large sample sizes and long durations of measurement.

Failure to see interactions of systolic BP, diastolic BP or hypertension with diabetes has been reported in the literature [7] and may have been related to the small sample of people with diabetes available to the analysis when stratified into controlled and uncontrolled groups or the fact that the majority of those with T2DM and hypertension were treated at wave 7 (87.7 and 88.4\%, respectively), and better control of T2DM and hypertension was achieved at wave 7 as opposed to wave $6(35.3 \%$ of those with diabetes were controlled at wave 6 and $56.3 \%$ were controlled at wave 7 ). The improved levels of control between waves 6 and 7 may be related to the fact that all subjects diagnosed with T2DM were referred to the metabolic unit at our affiliated hospital, State University of New York, Syracuse, NY, USA, or to their own physicians for treatment. While this procedure would lead to underestimated risk of higher PWV in the individuals in the study with diabetes and hypertension, it is an ethical requirement in most, if not all, longitudinal studies.

We have extended the cross-sectional work of Chirinos et al. [30] and others [31-33] by demonstrating prospective longitudinal associations between diabetes and PWV, with additional statistical control for other potential covariates in addition to age, sex and MAP, and in a sample of adults encompassing a wider age range. More recently, Xu et al. [34] calculated a genetic risk score and used a Mendelian randomization analysis and, on this basis, argued for a causal relationship between T2DM and arterial stiffening in a Chinese population. We did not have a genetic risk score in the present study, but we see a positive association between T2DM and PWV scores.

Given the value of PWV as an independent predictor of mortality and cardiovascular morbidity in subjects with diabetes [5, 35], studies of mechanisms linking T2DM with PWV are important [36]. Prenner and Chirinos [7] have reviewed mechanisms that may account for, or mediate, associations between T2DM and PWV with emphasis on the role of advanced glycation end-products and nitric oxide dysregulation. Briefly, oxidative stress may be responsible for the development of arterial stiffness in individuals with well-controlled T2DM [37, 38]. However, our findings were not attenuated with the addition of CRP to the model, and the CRP $\times$ T2DM interaction term was not significant. Other potential mechanisms underlying greater arterial stiffness in T2DM include aortic wall calcification, endothelial dysfunction, elevated extracellular matrix deposition of collagen and greater advanced glycation endproduct formation [39-41]. 
Among limitations, the study design was prospective but not longitudinal as no data on PWV were available in the MSLS database until wave 7 . Strengths of the study were that data were available on objectively measured cardiovascular risk factors in non-patients recruited from the community. The absence of HbA1c values is a limitation, but these were not considered essential to a diagnosis of diabetes when the study was conducted [42].

\section{Summary, Conclusions and Implications}

T2DM is prospectively associated with increased mean arterial stiffness (as inferred by PWV) over a 5-year period when adjusted for multiple cardiovascular risk factors and lifestyle- and PWV-related variables. Given the value of PWV as an independent predictor of mortality and cardiovascular morbidity in subjects with T2DM [5, 35], more studies are needed to improve the understanding of the mechanisms linking these factors and to direct strategies for individuals with diabetes to benefit arterial function [36]. The robust prediction of arterial stiffness over many years of life is observed even when diabetes mellitus and hypertension have been well controlled over time. Thus, prevention and early treatment of T2DM is critically important for the prevention or attenuation of arterial stiffness and attendant CVD mortality and morbidity.

\section{Acknowledgments}

We acknowledge Prof. David H.P. Streeten (deceased), formerly, Professor of Medicine (SUNY Health Science Center, Syracuse, NY, USA), for his collaborative work on diabetes and hypertension and making our work possible in terms of diagnoses and patient records, and his long-term collaborations with local physicians. We also wish to thank Prof. Steven Steinman at SUNY for his follow-on collaboration following Prof. Streeten's death.

\section{Disclosure Statement}

The authors have no conflicts of interest.

\section{Funding Sources}

The MSLS was supported by grants R01HL067358 and R01HL081290 from the National Heart, Lung and Blood Institute, National Institutes of Health (USA), and research grant R01AG03055 from the National Institute on Aging, National Institutes of Health (USA). G.E.C. is supported by a National Health and Medical Research Council (NHMRC) Sidney Sax Research Fellowship (GNT1054567) (Australia). The funding sources had no involvement in the study design, data collection, writing or decision to submit for publication.

\section{References}

1 Benetos A, Waeber B, Izzo J, Mitchell G, Resnick L, Asmar R, Safar M: Influence of age, risk factors, and cardiovascular and renal disease on arterial stiffness: clinical applications. Am J Hypertens 2002;15:1101-1108.

$\checkmark 2$ Boutouyrie P, Tropeano AI, Asmar R, Gautier I, Benetos A, Lacolley P, Laurent S: Aortic stiffness is an independent predictor of primary coronary events in hypertensive patients: a longitudinal study. Hypertension 2002;39:10-15.

3 Vlachopoulos C, Aznaouridis K, Stefanadis C: Prediction of cardiovascular events and all-cause mortality with arterial stiffness: a systematic review and meta-analysis. J Am Coll Cardiol 2010;55:1318-1327.

4 American Diabetes Association: Statistics about Diabetes. www.diabetes.org/diabetes-basics/statistics. 
Elias et al.: Associations between Type 2 Diabetes Mellitus and Arterial Stiffness: A Prospective Analysis Based on the Maine-Syracuse Study

5 Cruickshank K, Riste L, Anderson SG, Wright JS, Dunn G, Gosling RG: Aortic pulse-wave velocity and its relationship to mortality in diabetes and glucose intolerance: an integrated index of vascular function? Circulation 2002;106:2085-2090.

-6 Laurent S, Cockcroft J, Van Bortel L, Boutouyrie P, Giannattasio C, Hayoz D, Pannier B, Vlachopoulos C, Wilkinson I, Struijker-Boudier H: Expert consensus document on arterial stiffness: methodological issues and clinical applications. Eur Heart J 2006;27:2588-2605.

7 Prenner SB, Chirinos JA: Arterial stiffness in diabetes mellitus. Atherosclerosis 2015;238:370-379.

8 Mansour AS, Yannoutsos A, Majahalme N, Agnoletti D, Safar ME, Ouerdane S, Blacher J: Aortic stiffness and cardiovascular risk in type 2 diabetes. J Hypertens 2013;31:1584-1592.

-9 Cockcroft JR, Wilkinson IB, Evans M, McEwan P, Peters JR, Davies S, Scanlon MF, Currie CJ: Pulse pressure predicts cardiovascular risk in patients with type 2 diabetes mellitus. Am J Hypertens 2005;18:1463-1467; discussion 1468-1469.

10 van Dijk RA, Dekker JM, Nijpels G, Heine RJ, Bouter LM, Stehouwer CD: Brachial artery pulse pressure and common carotid artery diameter: mutually independent associations with mortality in subjects with a recent history of impaired glucose tolerance. Eur J Clin Invest 2001;31:756-763.

-11 Schram MT, Kostense PJ, Van Dijk RA, Dekker JM, Nijpels G, Bouter LM, Heine RJ, Stehouwer CD: Diabetes, pulse pressure and cardiovascular mortality: the Hoorn Study. J Hypertens 2002;20:1743-1751.

12 Johansen NB, Vistisen D, Rasmussen SSS, Wiinberg N, Borch-Johnsen K, Sandbaek A, Lauritzen T, Witte DR: The effect of changes in $\mathrm{HbA}(1 \mathrm{c})$ during 7 years on aortic stiffness in a population at high diabetes risk: ADDITION DK. Diabetologia 2011;54(suppl. 1):S70.

-13 Elias MF, Robbins MA, Budge MM, Abhayaratna WP, Dore GA, Elias PK: Arterial pulse wave velocity and cognition with advancing age. Hypertension 2009;53:668-673.

$\checkmark 14$ Elias PK, Elias MF, Robbins MA, Budge MM: Blood pressure-related cognitive decline: does age make a difference? Hypertension 2004;44:631-636.

-15 Elias PK, Elias MF, D’Agostino RB, Cupples LA, Wilson PW, Silbershatz H, Wolf PA: NIDDM and blood pressure as risk factors for poor cognitive performance. The Framingham Study. Diabetes Care 1997;20:1388-1395.

16 McKhann G, Drachman D, Folstein M, Katzman R, Price D, Stadlan EM: Clinical diagnosis of Alzheimer's disease: report of the NINCDS-ADRDA Work Group under the auspices of Department of Health and Human Services Task Force on Alzheimer's Disease. Neurology 1984;34:939-944.

17 World Health Organization: The ICD-10 Classification of Mental and Behavioural Disorders: Clinical Descriptions and Diagnostic Guidelines. Geneva, World Health Organization, 1992.

18 American Diabetes Association: Standards of medical care in diabetes - 2017. Diabetes Care 2017;40(suppl 1):S1-S132.

19 Kaaks R, Riboli E: Validation and calibration of dietary intake measurements in the EPIC project: methodological considerations. European Prospective Investigation into Cancer and Nutrition. Int J Epidemiol 1997; 26(suppl 1):S15-S25.

20 Riboli E, Kaaks R: The EPIC Project: rationale and study design. European Prospective Investigation into Cancer and Nutrition. Int J Epidemiol 1997;26(suppl 1):S6-S14.

-21 Wolf AM, Hunter DJ, Colditz GA, Manson JE, Stampfer MJ, Corsano KA, Rosner B, Kriska A, Willett WC: Reproducibility and validity of a self-administered physical-activity questionnaire. Int J Epidemiol 1994;23:991999.

-22 Elias MF, Robbins MA, Budge MM, Elias PK, Brennan SL, Johnston C, Nagy Z, Bates CJ: Homocysteine, folate, and vitamins B6 and B12 blood levels in relation to cognitive performance: the Maine-Syracuse study. Psychosom Med 2006;68:547-554.

23 Elias MF, Dore GA, Davey A, Abhayaratna WP, Goodell AL, Robbins MA: Norms and reference values for pulse wave velocity: one size does not fit all. JBM 2011, DOI: 10.5780/jbm2011.4.

24 Mancia G, De Backer G, Dominiczak A, Cifkova R, Fagard R, Germano G, Grassi G, Heagerty AM, Kjeldsen SE, Laurent S, et al: 2007 guidelines for the management of arterial hypertension: the Task Force for the Management of Arterial Hypertension of the European Society of Hypertension (ESH) and of the European Society of Cardiology (ESC). Eur Heart J 2007;28:1462-1536.

-25 Sakuragi S, Abhayaratna WP: Arterial stiffness: methods of measurement, physiologic determinants and prediction of cardiovascular outcomes. Int J Cardiol 2010;138:112-118.

-26 Laurent S, Boutouyrie P, Asmar R, Gautier I, Laloux B, Guize L, Ducimetiere P, Benetos A: Aortic stiffness is an independent predictor of all-cause and cardiovascular mortality in hypertensive patients. Hypertension 2001; 37:1236-1241.

-27 Smulyan H, Lieber A, Safar ME: Hypertension, diabetes type II, and their association: role of arterial stiffness. Am J Hypertens 2016;29:5-13.

-28 Agnoletti D, Mansour AS, Zhang Y, Protogerou AD, Ouerdane S, Blacher J, Safar ME: Clinical interaction between diabetes duration and aortic stiffness in type 2 diabetes mellitus. J Hum Hypertens 2017;31:189-194.

29 Agnoletti D, Millasseau SC, Topouchian J, Zhang Y, Safar ME, Blacher J: Pulse wave analysis with two tonometric devices: a comparison study. Physiol Meas 2014;35:1837-1848.

-30 Chirinos JA, Segers P, Gillebert TC, De Buyzere ML, Van Daele CM, Khan ZA, Khawar U, De Bacquer D, Rietzschel ER, Asklepios I: Central pulse pressure and its hemodynamic determinants in middle-aged adults with impaired fasting glucose and diabetes: the Asklepios study. Diabetes Care 2013;36:2359-2365. 
-31 Cameron JD, Bulpitt CJ, Pinto ES, Rajkumar C: The aging of elastic and muscular arteries: a comparison of diabetic and nondiabetic subjects. Diabetes Care 2003;26:2133-2138.

-32 Kimoto E, Shoji T, Shinohara K, Hatsuda S, Mori K, Fukumoto S, Koyama H, Emoto M, Okuno Y, Nishizawa Y: Regional arterial stiffness in patients with type 2 diabetes and chronic kidney disease. J Am Soc Nephrol 2006; 17:2245-2252.

33 Taniwaki H, Kawagishi T, Emoto M, Shoji T, Kanda H, Maekawa K, Nishizawa Y, Morii H: Correlation between the intima-media thickness of the carotid artery and aortic pulse-wave velocity in patients with type 2 diabetes. Vessel wall properties in type 2 diabetes. Diabetes Care 1999;22:1851-1857.

34 Xu M, Huang Y, Xie L, Peng K, Ding L, Lin L, Wang P, Hao M, Chen Y, Sun Y, et al: Diabetes and risk of arterial stiffness: a Mendelian randomization analysis. Diabetes 2016;65:1731-1740.

-35 Maeda Y, Inoguchi T, Etoh E, Kodama Y, Sasaki S, Sonoda N, Nawata H, Shimabukuro M, Takayanagi R: Brachialankle pulse wave velocity predicts all-cause mortality and cardiovascular events in patients with diabetes: the Kyushu Prevention Study of Atherosclerosis. Diabetes Care 2014;37:2383-2390.

-36 Petersen KS, Clifton PM, Lister N, Keogh JB: Effect of improving dietary quality on arterial stiffness in subjects with type 1 and type 2 diabetes: a 12 months randomised controlled trial. Nutrients 2016;8.

-37 Kotani K, Yamada T: Association between urinary 8-OHdG and pulse wave velocity in hypertensive patients with type 2 diabetes mellitus. Singapore Med J 2014;55:202-208.

-38 Zhang X, Liu JJ, Fang Sum C, Ying YL, Tavintharan S, Ng XW, Su C, Low S, Lee SB, Tang WE, et al: Central arterial stiffness is associated with systemic inflammation among Asians with type 2 diabetes. Diab Vasc Dis Res 2016; 13:303-306.

-39 Weber T: Arterial stiffness, wave reflections, and diabetes: a bidirectional relationship? Am J Hypertens 2010; 23:1047-1048.

-40 Odink AE, van der Lugt A, Hofman A, Hunink MG, Breteler MM, Krestin GP, Witteman JC: Risk factors for coronary, aortic arch and carotid calcification; the Rotterdam Study. J Hum Hypertens 2010;24:86-92.

-41 Zieman SJ, Melenovsky V, Kass DA: Mechanisms, pathophysiology, and therapy of arterial stiffness. Arterioscler Thromb Vasc Biol 2005;25:932-943.

-42 The International Expert Committee: International Expert Committee report on the role of the A1C assay in the diagnosis of diabetes. Diabetes Care 2009;32:1327-1334. 